\title{
Using Adaptive Replacement to Minimize Risk in the Oil and Gas Industry
}

\author{
Thomas Mazzuchi $^{1}$, Refik Soyer ${ }^{1}$, Neville Robinson $^{2}$, Khalid Aboura $^{3}$ \\ ${ }^{1}$ George Washington University, 20052, Washington D.C., U.S.A. \\ ${ }^{2}$ Flinders University, 5042, Bedford Park, Australia \\ ${ }^{3}$ American University of Armenia, 0019, Yerevan, Armenia
}

doi: https://doi.org/10.21467/abstracts.93.102

\begin{abstract}
ABST RA CT
Mazzuchi and Soyer (1996) presented a decision theoretic approach for determining optimal replacement strategies under replacement and repair scenarios. The Bayesian approach, adaptive in nature, takes into account failure and survival information at each planned replacement stage to update the optimal time until the next planned replacement. Under the assumption in which an item is replaced by a new one upon failure, the underlying process between two planned replacement times is a renewal process. The replacements upon failure that may occur between the planned replacement stages constitute renewals of the underlying renewal process. The times between renewals are the lifetimes of the items. Mazzuchi and Soyer (1996) made the Weibull assumption for the lifetime distribution of an item and used an approximation due to Smeitink and Dekker (1990) to compute the renewal function. Robinson and Aboura (1995, 2015) enhanced the adaptive approach by presenting a method for the exact calculation of the renewal function and its derivative due to Constantine and Robinson (1997). The method of finding zeros of a function, by Muller (1956) and Frank (1958), is adapted to the maintenance optimization problem, making use of the availability of the derivative of the renewal function. Robinson and Aboura (2015) made further improvements by providing a methodology for the assessment of the joint prior distribution of the parameters of the Weibull lifetime model. The prior distribution is determined through the specification of initial reliability estimates for different mission times. To provide a simple approach to carry out in practice, the authors developed an adaptive maintenance routine that uses the least squares estimates (LSE) and the maximum likelihood estimates (MLE). The approach retains the adaptive concept of Mazzuchi and Soyer (1996) but reduces the computational needs for averaging over the posterior distributions in the Bayesian approach.

In their paper, Robinson and Aboura (2015) provide an exact method, due to Constantine and Robinson (1997), for computing the Weibull renewalfunction and its derivativeh $(t / \lambda, \beta)=d H(t / \lambda, \beta) d t$. Another method due to Robinson (1997) can also be used to computethe renewal function to any desired degree of accuracy. That method solves directly the integral equation expressionfor $H(t / \lambda, \beta)$ (see e.g. Cox (1962) for general renewal theory) in terms of multi time-segment Chebyshev polynomial series.It is a method suitable for a wide range of probability density functions, both parametric and nonparametric. Thequicker method of Constantine and Robinson, dedicated to the Weibull renewal function was described in Robinson and Aboura $(1995,2015)$.Its extension to the Generalised Gamma renewal function is also available in Robinson (1997).

Van Noortwijk et al (1992) discuss topics in expert opinion in maintenance optimization based on considerable experience at Koninklijke/Shell Laboratorium, Amsterdam. Some of the adaptive models discussed in the above work were based on the scenarios found in the Oil and Gas Industry. The precise computation of statistical components such as the renewal function and its derivative, for a number of distributions including the Weibull and the Generalized Gamma can lead the way to the resolution of
\end{abstract}

(C) 2020 Copyright held by the author(s). Published by AIJR Publisher in "Abstracts of The Second Eurasian RISK-2020 Conference and Symposium" April 12- 19, 2020, Tbilisi, Georgia. Jointly organized by AMIR Technical Services LLC, Georgian Technical University, Institute of Geography (Kazakhstan) and Russian Institute of Petroleum Geology and Geophysics. 
The Second Eurasian RISK-2020 Conference and Symposium

important risk minimization problems in the industry. Our goal is to attend the conference for enlarging the set of tools available to the community, gathering important aspects in today's problems and discussing further the topic of assessing expert opinion in engineering environments.

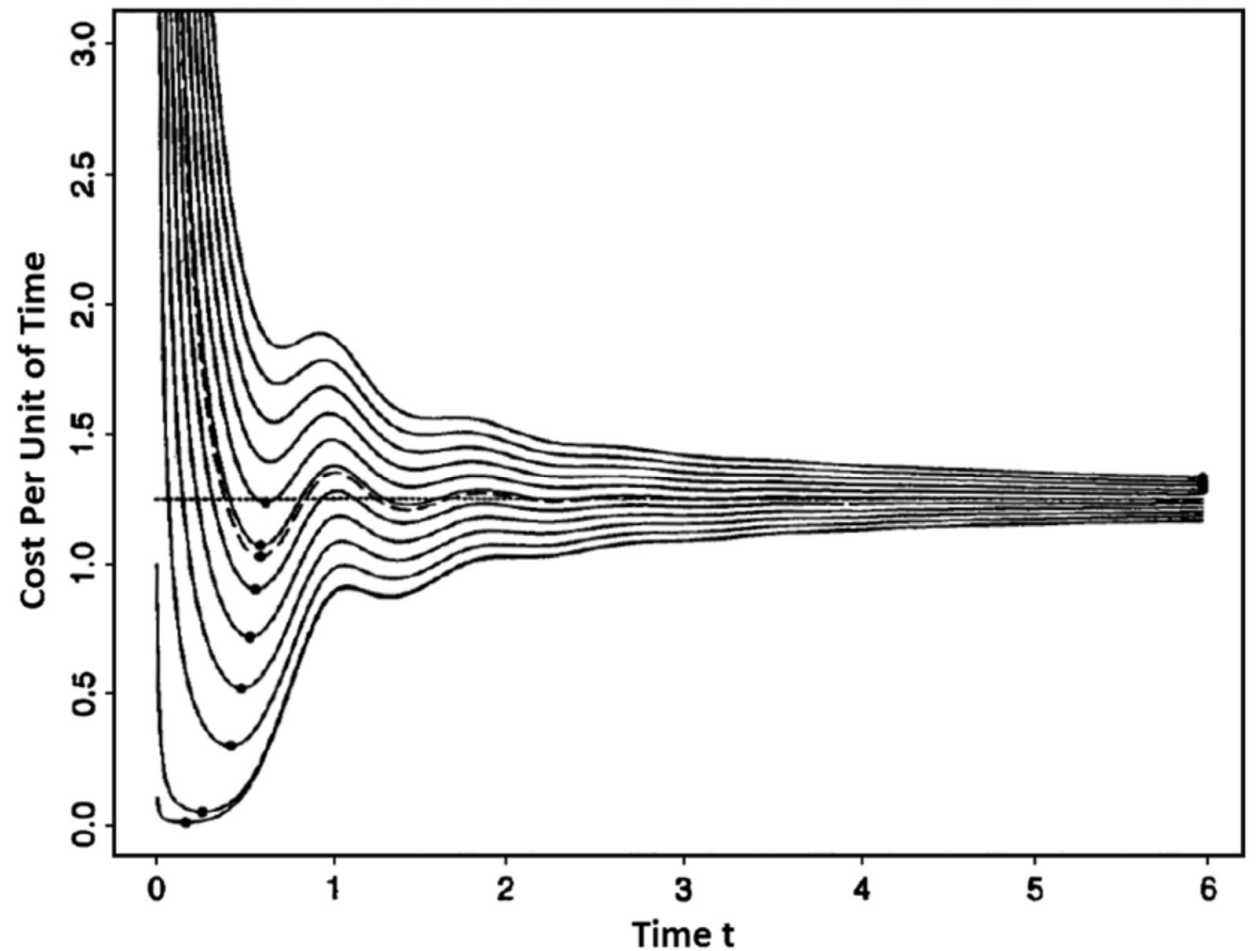

Figure 1. Maintenance cost function for different parameter values in Robinson and Aboura (2015)

\section{References}

1. Mazzuchi T, Soyer R. Adaptive Bayesian replacement strategies. Bayesian Statistics 5, Berger J, Bernardo J, David A, Smith A (eds). Oxford University Press: Oxford, 1996; 667-674.

2. Smeitink E, Dekker R. A simple approximation to the renewal function. IEEE Transactions on Reliability 1990; 39(1):71-75.

3. Robinson N, Aboura K. Optimal Replacement in a Renewal Process, The Commonwealth Scientific and Industrial Research Organisation (CSIRO) Report No. DMS-C/D 95/100, 1995.

4. Robinson N, Aboura K. Optimal Adaptive Replacement in a Renewal Process, Applied Stochastic Models in Business and Industry 2015; 31(4): 424-434.

5. Constantine A, Robinson N. The Weibull renewal function for moderate to large arguments. Computational Statistics \& Data Analysis 1997; 24:19-27.

6. Muller D. A method for solving algebraic equations using automatic computer. Mathematical Tables and Aids to Computation 1956; 10(56):208-215.

7. Frank W. Finding zeros of arbitrary functions. Journal of the Association for Computing Machinery 1958; 5(2):154-160.

8. Robinson N. Renewal functions as series. Communications in Statistics - Stochastic Models 1997; 13(3):577-604.

9. Cox D. Renewal Theory. Chapman-Hall: London, 1962.

10. Robinson N. Residue series for the generalised gamma renewal function. Computational techniques and applications: CTAC97, Noye J, Teubner M, Gill A (eds). World Scientific: Singapore, 1998; 591-598.

11. Van Noortwijk J. M, Dekker A, Cooke R, Mazzuchi T. Expert judgment in maintenance optimization, IEEE Transactions on Reliability; 41(3):427 - 432. 